\title{
ON LOG HODGE STRUCTURES OF HIGHER DIRECT IMAGES
}

\author{
TOSHIHARU MATSUBARA
}

\section{Introduction}

Let $Y$ be an analytic space endowed with an fs log structure $\mathscr{M}_{Y}$ in the sense of Fontaine-Illusie. The pair $\left(Y, \mathscr{M}_{Y}\right)$ is called an fs log analytic space (cf. $[\mathrm{KN}])$. For an fs $\log$ analytic space $\left(Y, \mathscr{M}_{Y}\right), \mathrm{K}$. Kato and C. Nakayama construct in $[\mathrm{KN}]$ a ringed space $\left(Y^{\log }, \mathcal{O}_{Y}^{\log }\right)$ endowed with a continuous surjective map $\tau: Y^{\log } \rightarrow Y$. In this paper, we mainly treat an object on $Y$ called a log Hodge structure which is defined by $\mathrm{K}$. Kato in [Ka2]. It consists of the following triplet that satisfies certain conditions (See 5.3):

- A sheaf of $\boldsymbol{Q}$-modules $\mathscr{H}_{\boldsymbol{Q}}$ on $Y^{\log }$.

- A sheaf of $\mathscr{O}_{Y}$-modules $\mathscr{H}_{\mathcal{O}}$ on $Y$ endowed with a descending filtration.

- An isomorphism of $\mathcal{O}_{Y}^{\log }$-modules $l: \mathscr{H}_{Q} \otimes_{Q} \mathcal{O}_{Y}^{\log } \cong \tau^{*} \mathscr{H}_{\mathcal{O}}$.

Let $f:\left(X, \mathscr{M}_{X}\right) \rightarrow\left(Y, \mathscr{M}_{Y}\right)$ be a morphism of fs log analytic spaces satisfying the following condition:

(*) Locally on $X$ and on $Y$,

(i) There exists a chart $P:=N \rightarrow \mathscr{M}_{Y}$, and a morphism of monoids

$$
P \rightarrow Q:=N^{r} ; \quad 1 \mapsto(1, \ldots, 1)
$$

for some $r \geq 1$, and

(ii) $X$ is isomorphic to an open subspace of $Y \times_{\operatorname{Spec} C[P]_{a n}}$ Spec $C[Q]_{a n}$, where Spec $C[P]$ and Spec $C[Q]$ are endowed with the log structures associated to $P \rightarrow C[P]$ and $Q \rightarrow C[Q]$, respectively.

First, we prove two basic properties.

THEOREM A. We have a quasi-isomorphism

$$
\left(f^{\log }\right)^{-1} \mathcal{O}_{Y}^{\log } \stackrel{\sim}{\longrightarrow} \omega_{X / Y}^{\bullet \log },
$$

where $\omega_{X / Y}^{1 \log }=\omega_{X / Y}^{1} \otimes_{\mathcal{O}_{X}} \mathcal{O}_{X}^{\log }$ and $\omega_{X / Y}^{\bullet \log }$ is its exterior algebra.

THEOREM B. Assume moreover $f$ is proper. Let $\tau: Y^{\log } \rightarrow Y$ be the canonical map. Then we have an isomorphism of $\mathcal{O}_{Y}^{\log }$-modules

$$
\imath: R^{m} f_{*}^{\log } \boldsymbol{Q} \otimes \mathcal{O}_{Y}^{\log } \cong \tau^{*} R^{m} f_{*} \omega_{X / Y}^{\bullet}
$$

for each $m$.

Received May 26, 1997; revised May 15, 1998. 
(**) For example, let $Y:=\{z \in C|| z \mid<1\}$ be the unit disk, and $f: X \rightarrow Y$ a projective surjective morphism of complex manifolds. We assume that $f$ is smooth over the punctured disk $Y^{*}=Y-\{0\}$ and that $X_{0}=f^{-1}(0)$ is a reduced divisor with normal crossings. Let $P \in X_{0}$. We assume that there exists a coordinate neighborhood $U$ of $P$ with coordinates $\left(z_{0}, \ldots, z_{n}\right)$ and an integer $r$ with $1 \leq r \leq n$ such that $P=(0, \ldots, 0)$ and $f \mid U\left(z_{1}, \ldots, z_{n}\right)=z_{1} \cdots z_{r}=z$. Let $\mathscr{M}_{Y}$ (resp. $\mathscr{M}_{X}$ ) be a sheaf of holomorphic functions on $Y$ (resp. $X$ ) which are invertible outside the origin (resp. $X_{0}$ ). Then we have a morphism $f:\left(X, \mathscr{M}_{X}\right) \rightarrow\left(Y, \mathscr{M}_{Y}\right)$ of fs log analytic spaces, which satisfies the condition (*).

If $f: X \rightarrow Y$ is a proper smooth morphism of complex manifolds, it is well known, as relative Poincaré lemma that $\Omega_{X / Y}^{*}$ is a resolution of the sheaf $f^{-1} \mathcal{O}_{Y}$. Using this, it is easy to construct an isomorphism of $\mathcal{O}_{Y}$-modules $R^{m} f_{*} \boldsymbol{Q} \otimes \mathcal{O}_{Y} \stackrel{\sim}{\rightarrow} R^{m} f_{*} \Omega_{X / Y}^{\bullet}$. Theorem $\mathrm{A}$ and Theorem $\mathrm{B}$ correspond to these facts. As for a log Hodge structure, we have

THEOREM C. Let $f: X \rightarrow Y$ be as in $(* *)$. Let $\mathscr{H}_{\mathbf{Q}}=\boldsymbol{R}^{m} f_{*}^{\log Q}, \mathscr{H}_{\mathcal{O}}=$ $R^{m} f_{*} \omega_{X / Y}^{\bullet}$ endowed with a filtration $R^{m} f_{*} \omega_{X / Y}^{\bullet \geq l}$ and $l$ the isomorphism as in Theorem $B$. Then the triplet $\left(\mathscr{H}_{\mathbf{Q}}, \mathscr{H}_{\mathcal{O}}, l\right)$ is a log Hodge structure on $Y$.

Here is some backgrounds. Let $Y:=\{z \in C|| z \mid<1\}$ be the unit disk, and $f: X \rightarrow Y$ a projective surjective morphism of complex manifolds. We assume that $f$ is smooth over the punctured disk $Y^{*}=Y-\{0\}$ and that $X_{0}=f^{-1}(0)$ is a divisor with normal crossings. We can consider a family of the polarized Hodge structures over $Y^{*}$. We can consider it as a holomorphic map from $Y^{*}$ to the classifying space of polarized Hodge structures modulo monodromy. This map is called the period map. W. Schmid has proved in [Sch] that the period map can be approximated by the associated nilpotent orbit. It is a holomorphic map from $Y$ to the compact dual of the classifying space of polarized Hodge structures, for which the origin of $Y$ is mapped to a polarized mixed Hodge structure. On the other hand, log geometry works well with varieties with normal crossings. The aim of Theorem $\mathrm{C}$ is to treat the above fact from a viewpoint of $\log$ geometry. In the proof of Theorem $\mathrm{C}$, we see that this $\log$ Hodge structure amounts to W. Schmid's nilpotent orbit theorem. We expect that log Hodge structures give a construction of compactification of some moduli space.

Remark 1.1. Related topics are studied by some people. S. Usui obtains a theorem corresponding to our Theorem B in [Usu] independently. His method is quite different from ours and he obtains a more general result. F. Kato also obtains Theorem A and Theorem B in [FKa]. His method is similar to ours.

In Section 2, we recall basic notions of a $\log$ geometry. In Section 3 , we prove Theorem A, a "log version" of relative Poincaré lemma. In Section 4, we prove Theorem B using the log Poincaré lemma and some inductions. In Section 5 , we define the log Hodge structure and prove Theorem $\mathrm{C}$. 
The author is grateful to Professor K. Kato for giving him the problem of this paper as well as the idea of log Hodge structures. The author is also grateful to Professors C. Nakayama and M. Somekawa for many discussions and suggestions to many improvements in this paper. He is also grateful to Professor T. Kajiwara for giving him some advice on the presentation.

\section{The ringed space $\left(X^{\log }, \mathcal{O}_{X}^{\log }\right)$ associated to a log scheme $X$}

In this section, we recall some notions in log geometry, which will be used in the later sections. For more systematic descriptions, see [Kal], [KN].

DEFINITION 2.1. Let $\stackrel{\circ}{X}$ be an analytic space and $\mathcal{O}_{\dot{X}}$ the sheaf of holomorphic functions on $\stackrel{X}{X}$. A pre-log structure on $\dot{X}$ is a sheaf of monoids $\mathscr{M}$ on $\dot{X}$ endowed with a homomorphism of sheaves of monoids $\alpha: \mathscr{M} \rightarrow \mathcal{O}_{\dot{X}}$ with respect to the multiplication on $\mathcal{O}_{\dot{X}}$. It is denoted by $(\mathscr{M}, \alpha)$, or simply $\mathscr{M}$. A pre-log structure is said to be a $\log$ structure if $\alpha^{-1}\left(\mathcal{O}_{\grave{X}}^{*}\right) \rightarrow \mathcal{O}_{\grave{X}}^{*}$ is an isomorphism via $\alpha$.

2.2. A log analytic space $X$ is a pair of an analytic space ${ }^{\circ}$ and a $\log$ structure $\mathscr{M}_{X}$ on $\stackrel{\odot}{X}$. It is denoted by $X:=\left(\stackrel{\circ}{X}, \mathscr{M}_{X}\right)$, or simply by $\left(X, \mathscr{M}_{X}\right)$. A morphism $\left(X, \mathscr{M}_{X}\right) \rightarrow\left(Y, \mathscr{M}_{Y}\right)$ of log analytic spaces is defined to be a pair of a morphism of analytic spaces $f: X \rightarrow Y$ and a homomorphism $h: f^{-1}\left(\mathscr{M}_{Y}\right) \rightarrow$ $\mathscr{M}_{X}$ such that the diagram

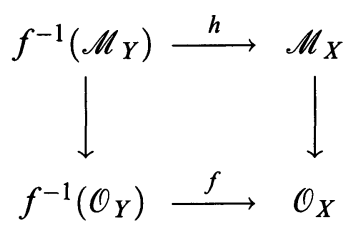

is commutative. It is denoted by $(f, h)$, or simply by $f$.

2.3. For a pre-log structure $(\mathscr{M}, \alpha)$ on $X$, its associated log structure $\mathscr{M}^{a}$ is defined to be the push out of

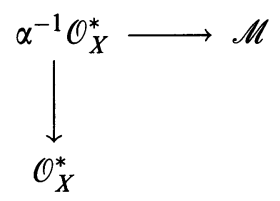

in the category of sheaves of monoids, endowed with the homomorphism

$$
\mathscr{M}^{a} \rightarrow \mathcal{O}_{X} ; \quad(a, b) \mapsto \alpha(a) b \quad\left(a \in \mathscr{M}, b \in \mathcal{O}_{X}^{*}\right) .
$$

2.4. A monoid $P$ is said to be an fs monoid if it satisfies the following three conditions:

(i) $P$ is finitely generated.

(ii) If $a, b, c \in P$ and $a b=a c$, then $b=c$. 
(iii) If $a \in P^{\mathrm{gp}}$ and $a^{n} \in P$ for some $n \neq 1$, then $a \in P$. Here $P^{\mathrm{gp}}$ is the group associated to $P$.

2.5. A $\log$ analytic space $\left(X, \mathscr{M}_{X}\right)$ is said to be an fs $\log$ analytic space if locally there exists a constant sheaf $P$ of fs monoids and a homomorphism $P \rightarrow \mathcal{O}_{X}$ such that the $\log$ structure $\mathscr{M}_{X}$ is isomorphic to the log structure associated to the pre-log structure defined by $P$. A pair of $P$ and the canonical map $P \rightarrow \mathscr{M}_{X}$ is called a chart. By definition, a chart exists locally.

Definition 2.6. Let $X:=\left(\stackrel{\circ}{X}, \mathscr{M}_{X}\right)$ be an fs log analytic space. We define the associated topological space $X^{\log }$ in the following way. Let $\stackrel{\circ}{T}$ be the analytic space Spec $C$ endowed with $\log$ structure $\mathscr{M}_{T}$ given by

$$
\Gamma\left(\stackrel{\circ}{T}, \mathscr{M}_{T}\right)=\boldsymbol{R}_{\geq 0} \times \mathbf{S}^{1}
$$

where

$$
\boldsymbol{R}_{\geq 0}=\{x \in \boldsymbol{R} ; x \geq 0\} \text { and } \mathbf{S}^{1}=\{x \in \boldsymbol{C} ;|x|=1\}
$$

are considered as the multiplicative semi-groups and the morphism $\mathscr{M}_{T} \rightarrow \mathcal{O}_{T}$ is given by

$$
\boldsymbol{R}_{\geq 0} \times \mathbf{S}^{1} \longrightarrow \boldsymbol{C} ; \quad(x, y) \mapsto x y .
$$

Let $T$ be the $\log$ analytic space $\left(\stackrel{\circ}{T}, \mathscr{M}_{T}\right)$. As a set, we define $X^{\log }$ to be the set of all morphisms $T \rightarrow X$ of $\log$ analytic spaces over $C$. We have the canonical surjective map $\tau: X^{\log } \rightarrow X$. We define the topology of $X^{\log }$ as follows. Working on locally on $X$, let $\alpha: P \rightarrow M_{X}$ be a chart of $\mathscr{M}_{X}$. Then, by using the homomorphism $P^{\mathrm{gp}} \rightarrow \mathscr{M}_{X}^{\mathrm{gp}}, X^{\log }$ is identified with a closed subset of $X \times \operatorname{Hom}\left(P^{\mathrm{gp}}, \mathbf{S}^{1}\right)$. The topology of $X^{\log }$ is given by this identification.

Lemma $2.7(\mathrm{KN},(1.3)) . \quad(\mathrm{i})$ The map $\tau: X^{\log } \rightarrow \stackrel{\circ}{X}$ is continuous. Furthermore it is proper, that is, for any compact subset $C$ of $X$, the subspace $\tau^{-1}(C)$ of $X^{\log }$ is compact.

(ii) For $x \in \stackrel{\circ}{X}, \tau^{-1}(x)$ is homeomorphic to the product of $r$ copies of $\mathbf{S}^{1}$ where $r$ is the rank of $\mathscr{M}_{X, x}^{\mathrm{gp}} / \mathcal{O}_{X, x}^{*}$.

(iii) Let $X:=\left(\stackrel{\circ}{X}, \mathscr{M}_{X}\right)$ and $Y:=\left(\stackrel{\circ}{Y}, \mathscr{M}_{Y}\right)$ be $f_{s}$ log analytic spaces, respectively. Let $f: X \rightarrow Y$ be a morphism of log analytic spaces. Assume $f^{-1} \mathscr{M}_{Y} \stackrel{\sim}{\rightarrow} \mathscr{M}_{X}$. Then the diagram of topological spaces

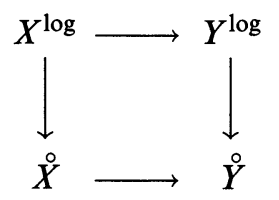

is cartesian. 
2.8. Let $\left(X, \mathscr{M}_{X}\right)$ be an fs $\log$ analytic space and $\tau: X^{\log } \rightarrow X$ the canonical map. For a topological space $A$, we denote by $\operatorname{Cont}(, A)$ the sheaf of continuous functions on $X^{\log }$ with values in $A$. Let $\tau^{-1}\left(\mathscr{M}_{X}^{\mathrm{gp}}\right) \rightarrow \operatorname{Cont}\left(, S^{\mathbf{l}}\right)$ be the natural map. Let $\operatorname{Cont}(, \boldsymbol{R}) \rightarrow \operatorname{Cont}\left(, \boldsymbol{S}^{\mathbf{l}}\right)$ be the map given by composition with exp. We define a sheaf $\mathscr{L}$ of abelian groups on $X^{\log }$ to be the fibre product of $\operatorname{Cont}(, i \boldsymbol{R})$ and $\tau^{-1} \mathscr{M}_{X}^{\mathrm{gp}}$ over $\operatorname{Cont}\left(, \boldsymbol{S}^{\mathbf{1}}\right)$. Let $h: \tau^{-1} \mathcal{O}_{X} \rightarrow \mathscr{L}$ be the map induced by the map $\tau^{-1} \mathcal{O}_{X} \rightarrow \operatorname{Cont}(, \boldsymbol{R}) ; f \mapsto f-\operatorname{Re}(f)$. Then we have the following commutative diagram with exact rows.

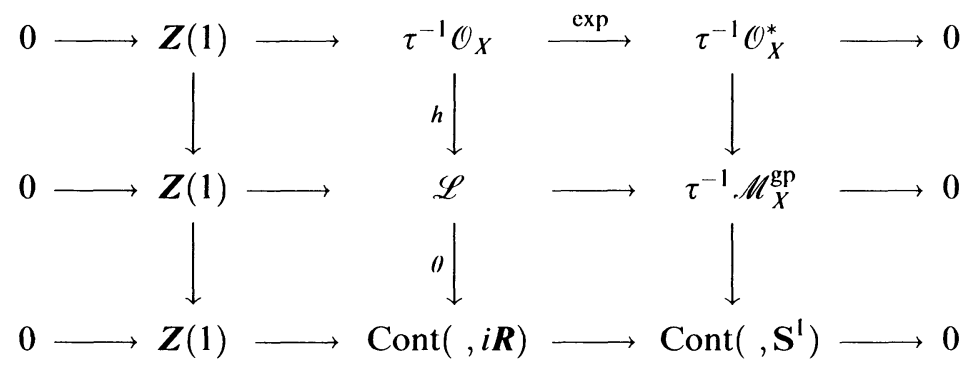

Definition 2.9. Let $\operatorname{Sym}_{Z}(\mathscr{L})$ be the symmetric algebras of $\mathscr{L}$ over $\boldsymbol{Z}$. We define a sheaf $\mathcal{O}_{X}^{\log }$ of $\tau^{-1} \mathcal{O}_{X}$-algebras on $X^{\log }$ as follows:

$$
\mathcal{O}_{X}^{\log }=\left(\tau^{-1} \mathcal{O}_{X} \otimes_{Z} \operatorname{Sym}_{Z}(\mathscr{L})\right) / \mathfrak{a}
$$

where $\boldsymbol{a}$ is the ideal of $\tau^{-1} \mathcal{O}_{X} \otimes_{Z} \operatorname{Sym}_{Z}(\mathscr{L})$ generated by local sections of the form

$$
f \otimes 1-1 \otimes h(f) \text { for } f \in \tau^{-1} \mathcal{O}_{X} .
$$

For $r \in Z$, we define a filtration $\operatorname{fil}_{r}\left(\mathcal{O}_{X}^{\log }\right)$ of $\mathcal{O}_{X}^{\log }$ to be the image of $\tau^{-1} \mathcal{O}_{X} \otimes_{Z} \oplus_{l=0}^{r} \operatorname{Sym}_{Z}^{l} \mathscr{L}$ in $\mathcal{O}_{X}^{\log }$, where $\operatorname{Sym}_{Z}^{l} \mathscr{L}$ denotes the $i$-th symmetric power over $Z$.

Lemma $2.10(\mathrm{KN},(3.3))$. Let $x$ be a point of $X, y$ a point of $\tau^{-1}(x) \subseteq X^{\log }$ and $\left(t_{i}\right)_{1 \leq ı \leq n}$ a family of elements of the stalk $\mathscr{L}_{y}$ whose image under exp is a $Z$-basis of $\left(\mathscr{M}_{X}^{\mathrm{gp}} / \mathcal{O}_{X}^{*}\right)_{X}$. Then the $\mathcal{O}_{X, x}$-algebra homomorphism

$$
\mathcal{O}_{X, x}\left[T_{1}, \ldots, T_{n}\right] \stackrel{\sim}{\rightarrow} \mathcal{O}_{X, y}^{\log } ; \quad T_{\iota} \mapsto t_{i}
$$

is an isomorphism.

LemMA $2.11(\mathrm{KN}, 3.4))$. (i) fil $_{0}\left(\mathcal{O}_{X}^{\log }\right)=\tau^{-1} \mathcal{O}_{X}$.

(ii) The canonical homomorphism $\tau^{-1} \mathscr{M}_{X}^{\mathrm{gp}} \cong \mathscr{L} / Z(1) \rightarrow \mathrm{fil}_{1}\left(\mathcal{O}_{X}^{\log }\right) / \tau^{-1} \mathcal{O}_{X}$ induces an isomorphism

$$
\tau^{-1} \mathcal{O}_{X} \otimes{ }_{Z} \tau^{-1}\left(\operatorname{Sym}_{Z}^{r}\left(\mathscr{M}_{X}^{\mathrm{gp}} / \mathcal{O}_{X}^{*}\right)\right) \cong \operatorname{fil}_{r}\left(\mathcal{O}_{X}^{\log }\right) / \mathrm{fil}_{r-1}\left(\mathcal{O}_{X}^{\log }\right)
$$

for any $r \geq 0$. 


\section{Logarithmic relative Poincaré lemma}

The aim of this section is to prove Theorem A.

Proposition 3.1 (Relative Poincaré lemma). Let $f: X \rightarrow Y$ be a smooth holomorphic map of complex manifolds. Then

$$
f^{-1} \mathcal{O}_{Y} \rightarrow \Omega_{X / Y}^{\bullet}
$$

is a quasi-isomorphism.

3.2. Let $f:\left(X, \mathscr{M}_{X}\right) \rightarrow\left(Y, \mathscr{M}_{Y}\right)$ be a morphism of fs log analytic spaces satisfying the following conditions:

Locally on $X$ and on $Y$,

(i) there exists a chart $P:=N \rightarrow \mathscr{M}_{Y}$, and a morphism of monoids

$$
P \rightarrow Q:=N^{r} ; \quad 1 \mapsto(1, \ldots, 1),
$$

for some $r \geq 1$, and

(ii) $X$ is isomorphic to an open subspace of $Y \times_{\operatorname{Spec} C[P]_{a n}} \operatorname{Spec} C[Q]_{a n}$, where Spec $C[P]$ and Spec $C[Q]$ are endowed with log structures associated to $P \rightarrow C[P]$ and $Q \rightarrow C[Q]$, respectively.

Proposition 3.3. Let $Q$ be the monoid $N$ and $P$ the monoid $N^{r}$ for $r \in N$. We denote $i$-th basis of $P$ as $e_{i}$. Let $X$ be the analytic space $C^{r}$ and $Y$ the analytic space $C$. Let $\left(t_{1}, \ldots, t_{r}\right)$ (resp. z) be a coordinate of $X$ (resp. Y). Let $f: X \rightarrow Y$ be a morphism defined by $\left(t_{1}, \ldots, t_{r}\right) \mapsto t_{1} \cdots t_{r}$. Let $\alpha$ (resp. $\left.\beta\right)$ be the morphism of monoids $P \rightarrow \Gamma\left(X, \mathcal{O}_{X}\right)$ (resp. $\left.Q \rightarrow \Gamma\left(Y, \mathcal{O}_{Y}\right)\right)$ defined by $n \cdot e_{i} \mapsto t_{n}^{n}$ (resp. $\left.n \mapsto z^{n}\right)$. Let $\left(X, \mathscr{M}_{X}\right)$ and $\left(Y, \mathscr{M}_{Y}\right)$ be the associated log analytic spaces, respectively. Then we have an isomorphism of $f^{-1} \mathcal{O}_{Y}$-modules

$$
\xi_{1}: f^{-1} \mathcal{O}_{Y} \otimes_{Z} \bigwedge^{q} \frac{\left(\mathscr{M}_{X}^{\mathrm{gp}} / \mathcal{O}_{X}^{*}\right)}{f^{-1}\left(\mathscr{M}_{Y}^{\mathrm{gp}} / \mathcal{O}_{Y}^{*}\right)} \stackrel{\sim}{\rightarrow} \mathscr{H}^{q}\left(\omega_{X / Y}^{*}\right) \quad \text { for all } q \geq 0 .
$$

Proof. If $\mathscr{Z}^{q} \subset \omega_{X / Y}^{q}$ is a sheaf of sections of cocycles, we have a morphism

$$
f^{-1} \mathcal{O}_{Y} \otimes \bigwedge^{q}\left(\mathscr{M}_{X}^{\mathrm{gp}} / f^{-1} \mathscr{M}_{Y}^{\mathrm{gp}}\right) \rightarrow \mathscr{Z}^{q} ; \quad a \otimes \wedge_{l} b_{i} \mapsto a \wedge_{l} d \log \left(b_{i}\right) .
$$

Let $z$ be a point of $X$. If $b_{i} \in \mathcal{O}_{X, z}^{*}$ for some $i$, then a branch of $\log b_{i}$ is in $\mathcal{O}_{X, z}^{*}$ and hence the image of $a \otimes \wedge_{l} b_{i}$ is a coboundary. Hence we have a well-defined morphism of sheaves

$$
\xi_{1}: f^{-1} \mathcal{O}_{Y} \otimes \bigwedge^{q} \frac{\mathscr{M}_{X}^{\mathrm{gp}} / \mathcal{O}_{X}^{*}}{f^{-1}\left(\mathscr{M}_{Y}^{\mathrm{gp}} / \mathcal{O}_{Y}^{*}\right)} \rightarrow \mathscr{H}^{q}\left(\omega_{X / Y}^{\bullet}\right) .
$$

It is enough to prove that $\xi_{1}$ is an isomorphism at each stalks.

CASE 1: Let $x=\left(t_{1}, \ldots, t_{r}\right)$ be a point of $X$ such that $t_{1} \cdots t_{r} \neq 0$. Since its $\log$ structure is trivial on a neighborhood of $x$, the stalk at $x$ of the right hand 
LOG HODGE STRUCTURES

87

side of (3.4) is $f^{-1} \mathcal{O}_{Y, x}$ (resp. 0$)$ if $q=0$ (resp. if $q \neq 0$ ). Hence we obtain the desired isomorphism in this case by 3.1.

CASE 2: Let $x=(0,0, \ldots, 0)$. We can compute the right hand side of (3.4) as $f^{-1} \mathcal{O}_{Y, x} \otimes_{Z} \wedge^{q} \boldsymbol{Z}^{r} / \boldsymbol{Z}$. We will prove in three steps that

$$
\mathscr{H}^{q}\left(\omega_{X / Y}^{\bullet}\right)_{x}=\left\{\sum_{1 \leq l_{1}<\cdots<l_{q} \leq r} a_{i_{1}, \ldots, l_{q}} f_{l_{1}} \wedge \cdots \wedge f_{i_{q}} ; a_{i_{1}, \ldots, l_{q}} \in f^{-1} \mathcal{O}_{Y, x}\right\} / I,
$$

where $f_{i}=d t_{i} / t_{i}(1 \leq i \leq r)$ and $I$ is the submodule generated by $f_{1}+\cdots+f_{r}$. We have

$$
\omega_{X / Y, x}^{1}=\frac{\mathcal{O}_{X, x} f_{1} \oplus \cdots \oplus \mathcal{O}_{X, x} f_{r}}{\mathcal{O}_{X, x}\left(f_{1} \oplus \cdots \oplus f_{r}\right)} .
$$

We can write an element of $\omega_{X / Y, x}^{q}$ as

$$
\sum_{e_{1}, \ldots, e_{r}}\left(\sum_{2 \leq l_{1}<\cdots<l_{q} \leq r} a_{i_{1}, \ldots, l_{q}}^{e_{1}, \ldots, e_{r}} f_{i_{1}} \wedge \cdots \wedge f_{i_{q}}\right) t_{1}^{e_{1}} \cdots t_{r}^{e_{r}}, \quad a_{i_{1}, \ldots, l_{q}}^{e_{1}, \ldots, e_{r}} \in C .
$$

Let $M_{e_{1}, \ldots, e_{r}}^{q}$ be the submodule

$$
\left\{\left(\sum_{2 \leq i_{1}<\cdots<l_{q} \leq r} a_{i_{1}, \ldots, l_{q}} f_{i_{1}} \wedge \cdots \wedge f_{i_{q}}\right) t_{1}^{e_{1}} \cdots t_{r}^{e_{r}} ; a_{i_{1}, \ldots, l_{q}} \in C\right\}
$$

of $\omega_{X / Y, x}^{q}$. Then we have

$$
\omega_{X / Y, x}^{q}=\left\{\phi \in \sum_{e_{1}, \ldots, e_{r}} M_{e_{1}, \ldots, e_{r}}^{q} ; \phi \text { converges }\right\} .
$$

STEP 1. For $\phi \in M_{e_{1}, \ldots, e_{r}}^{q}$, we will prove that $d \phi \in M_{e_{1}, \ldots, e_{r}}^{q+1}$.

We write $\phi$ as follows:

$$
\phi=\left(\sum_{2 \leq l_{1}<\cdots<l_{q} \leq r} a_{i_{1}, \ldots, l_{q}} f_{i_{1}} \wedge \cdots \wedge f_{i_{q}}\right) t_{1}^{e_{1}} \cdots t_{r}^{e_{r}} .
$$

We have

$$
\begin{aligned}
d \phi & =t_{1}^{e_{1}} \cdots t_{r}^{e_{r}} \sum_{2 \leq l_{1}<\cdots<l_{q} \leq r} \sum_{j \notin\left\{l_{1}, \ldots, l_{q}\right\}} a_{i_{1}, \ldots, l_{q}} e_{j} f_{j} \wedge f_{i_{1}} \wedge \cdots \wedge f_{i_{q}} \\
& =t_{1}^{e_{1}} \cdots t_{r}^{e_{r}} \sum_{2 \leq l_{1}<\cdots<l_{q} \leq r} \sum_{j \notin\left\{1, l_{1}, \ldots, l_{q}\right\}} a_{i_{1}, \ldots, l_{q}}\left(e_{j}-e_{1}\right) f_{j} \wedge f_{i_{1}} \wedge \cdots \wedge f_{i_{q}} \\
& =t_{1}^{e_{1}} \cdots t_{r}^{e_{r}} \sum_{2 \leq l_{1}<\cdots<l_{q+1} \leq r}\left\{\sum_{k=1}^{q+1}(-1)^{k-1} a_{i_{1}, \ldots, \hat{l}_{k}, \ldots, l_{q+1}}\left(e_{i_{k}}-e_{1}\right)\right\} f_{i_{1}} \wedge \cdots \wedge f_{i_{q+1}} .
\end{aligned}
$$


STEP 2. Let $\phi \in M_{e_{1}, \ldots, e_{r}}^{q} \cap \operatorname{ker} d$. We will prove that $\phi \notin \operatorname{im} d$ if and only if $e_{1}=\cdots=e_{r}$ and $\phi \neq 0$.

Let

$$
\psi=\left(\sum_{2 \leq t_{1}<\cdots<i_{q-1} \leq r} \tilde{a}_{i_{1}, \ldots, l_{q-1}} f_{i_{1}} \wedge \cdots \wedge f_{i_{q-1}}\right) t_{1}^{e_{1}} \cdots t_{r}^{e_{r}}
$$

be an element of $M_{e_{1}, \ldots, e_{r}}^{q-1}$. Then $\phi \in$ im $d$ if and only if there exists a complex vector $\left(\tilde{a}_{i_{1}, \ldots, l_{q-1}}\right)$ such that $\phi=d \psi$. This is translated as what the simultaneous linear equations in the $\tilde{a}_{i_{1}, \ldots, \hat{l}_{k}, \ldots I_{q}}$

$$
\left\{\begin{array}{r}
\sum_{k=1}^{q}(-1)^{k-1} \tilde{a}_{i_{1}, \ldots, \hat{i}_{k}, \ldots, l_{q}}\left(e_{i_{k}}-e_{1}\right)=a_{i_{1}, \ldots, i_{q}} \\
\left(2 \leq i_{1}<\cdots<i_{q} \leq r\right)
\end{array}\right.
$$

has a solution. "If part" is clear. In order to prove "only if part", we may assume that $e_{1} \neq e_{2}$ without loss of generality. Let $\tilde{a}$ (resp. $a$ ) be the vector $\left(\tilde{a}_{i_{1}, \ldots, \hat{i}_{k}, \ldots, l_{q}}\right)$ (resp. $\left.\left(a_{i_{1}, \ldots, l_{q}}\right)\right)$ and $A$ the matrix whose entries consist of coefficients of the simultaneous equations (3.5). For $I=\left\{i_{1}, \ldots, i_{q}\right\}$, we call the equation

$$
\sum_{k=1}^{q}(-1)^{k-1} \tilde{a}_{i_{1}, \ldots, \hat{i}_{k}, \ldots, l_{q}}\left(e_{i_{k}}-e_{1}\right)=a_{i_{1}, \ldots, l_{q}}
$$

as an $I$-th equation. We call the row of the matrix $A$ corresponding to the $I$-th equation as $I$-th row. Then there exists a solution of (3.5) if and only if rank $A=\operatorname{rank}(A, a)$. Let $I$ be a set $\left\{i_{1}, \ldots, i_{q}\right\}$ such that $2<i_{1}<\cdots<i_{q} \leq r$. For each $k=1, \ldots, q$, we denote $J_{k}:=\{2\} \cup\left(I-\left\{i_{k}\right\}\right)$. We will make an elementary transformation as follows. Multiply the $I$-th row of $A$ by $e_{2}-e_{1}$, and add $(-1)^{k}\left(e_{i_{k}}-e_{1}\right)$ times the $J_{k}$-th row of $A$ to it for all $k$. Then we have

$$
\begin{aligned}
& (I \text {-th equation }) \times\left(e_{2}-e_{1}\right)+\sum_{k=1}^{q}\left((-1)^{k}\left(J_{k} \text {-th equation }\right)\right) \times\left(e_{i_{k}}-e_{1}\right) \\
& =\sum_{k=1}^{q}(-1)^{k-1} \tilde{a}_{i_{1}, \ldots, \hat{k}_{k}, \ldots, l_{q+1}}\left(e_{i_{k}}-e_{1}\right)\left(e_{2}-e_{1}\right) \\
& \quad+\sum_{k=1}^{q}\left\{(-1)^{k} \tilde{a}_{i_{1}, \ldots, \hat{l}_{k}, \ldots, l_{q+1}}\left(e_{2}-e_{1}\right)\left(e_{i_{k}}-e_{1}\right)\right. \\
& \quad+\sum_{l=1}^{k-1}(-1)^{l+k} \tilde{a}_{2, l_{1}, \ldots, l_{l-1}, \hat{l}_{l}, l_{l+1}, \ldots, l_{k-1}, \hat{l}_{k}, l_{k+1}, \ldots, l_{q}}\left(e_{i_{l}}-e_{1}\right)\left(e_{i_{k}}-e_{1}\right) \\
& \left.\quad+\sum_{l=k+1}^{q}(-1)^{l+k-1} \tilde{a}_{2, l_{1}, \ldots, l_{k-1}, \hat{l}_{k}, l_{k+1}, \ldots, l_{l-1}, \hat{l}_{l}, l_{l+1}, \ldots, l_{q}}\left(e_{i_{l}}-e_{1}\right)\left(e_{i_{k}}-e_{1}\right)\right\}
\end{aligned}
$$




$$
\begin{aligned}
= & \sum_{1 \leq l<k \leq q}(-1)^{l+k} \tilde{a}_{2, l_{1}, \ldots, l_{l-1}, \hat{l}_{l}, l_{l+1}, \ldots, l_{k-1}, \hat{\iota}_{k}, l_{k+1}, \ldots, l_{q}}\left(e_{i_{l}}-e_{1}\right)\left(e_{i_{k}}-e_{1}\right) \\
& +\sum_{1 \leq l<k \leq q}(-1)^{l+k-1} \tilde{a}_{2, l_{1}, \ldots, l_{l-1}, \hat{l}_{l}, l_{l+1}, \ldots, l_{k-1}, \hat{l}_{k}, l_{k+1}, \ldots, l_{q}}\left(e_{i_{l}}-e_{1}\right)\left(e_{i_{k}}-e_{1}\right) \\
= & 0 .
\end{aligned}
$$

This means that, by the above elementary transformation, $A$ is transformed to

$$
\left(\begin{array}{ccc|c}
e_{2}-e_{1} & & 0 & \\
& \ddots & & * \\
0 & & e_{2}-e_{1} & \\
\hline & 0 & & 0
\end{array}\right)
$$

Using the same elementary transformation for the vector $a$, its $I$-th row is transformed to

$$
\sum_{k=0}^{q}(-1)^{k} a_{i_{0}, \ldots, \hat{i}_{k}, \ldots, l_{q}}\left(e_{i_{k}}-e_{1}\right), \quad\left(i_{0}=2\right) .
$$

Therefore the condition $\phi \in \operatorname{ker} d$ implies that (3.6) is equal to 0 . This means that $(A, a)$ is transformed to

$$
\left(\begin{array}{ccc|c|c}
e_{2}-e_{1} & & 0 & & \\
& \ddots & & * & * \\
0 & & e_{2}-e_{1} & & \\
\hline & 0 & & 0 & 0
\end{array}\right)
$$

Thus we have $\operatorname{rank} A=\operatorname{rank}(A, a)$ as desired.

STEP 3. Let $\phi$ be an element of $\omega_{X / Y, x}^{q} \cap \operatorname{ker} d$ and $\phi^{e_{1}, \ldots, e_{r}}$ an element of $M_{e_{1}, \ldots, e_{r}}^{q}$ such that $\sum_{e_{1}, \ldots, e_{r}} \phi^{e_{1}, \ldots, e_{r}}$ converges in some neighborhood of $x$ and such that this sum is equal to $\phi$. We claim that there exists $\psi \in \omega_{X / Y, x}^{q-1}$ such that $d \psi=\phi$ if and only if there exist $\psi^{e_{1}, \ldots, e_{r}} \in M_{e_{1}, \ldots, e_{r}}^{q-1}$ such that $d \psi^{e_{1}, \ldots, e_{r}}=\phi^{e_{1}, \ldots, e_{r}}$ for all $\left(e_{1}, \ldots, e_{r}\right)$.

The "only if part" is clear, hence we will prove the "if part." All what we have to do is to prove $\sum_{e_{1}, \ldots, e_{r}} \psi^{e_{1}, \ldots, e_{r}}$ also converges in some neighborhood of $x$. We write $\phi^{e_{1}, \ldots, e_{r}}\left(\right.$ resp. $\left.\psi^{e_{1}, \ldots, e_{r}}\right)$ as

$$
\begin{gathered}
\left(\sum_{2 \leq l_{1}<\cdots<l_{q} \leq r} a_{i_{1}, \ldots, l_{q}}^{e_{1}, \ldots, e_{r}} f_{i_{1}} \wedge \cdots \wedge f_{i_{q}}\right) t_{1}^{e_{1}} \cdots t_{r}^{e_{r}} \\
\left(\operatorname{resp} \cdot\left(\sum_{2 \leq l_{1}<\cdots<i_{q-1} \leq r} \tilde{a}_{i_{1}, \ldots, l_{q-1}}^{e_{1}, \ldots, e_{r}} f_{i_{1}} \wedge \cdots \wedge f_{i_{q-1}}\right) t_{1}^{e_{1}} \cdots t_{r}^{e_{r}}\right) .
\end{gathered}
$$


Then it is enough to prove that

$$
\sum_{e_{1}, \ldots, e_{r}} \tilde{a}_{i_{1}, \ldots, l_{q-1}}^{e_{1}, \ldots, e_{r}} t_{1}^{e_{1}} \cdots t_{r}^{e_{r}}
$$

converges in some neighborhood of $x$ for all $\left(i_{1}, \ldots, i_{q-1}\right)$. By the argument in Step 2, we can solve the simultaneous linear equations in $\tilde{a}_{i_{1}, \ldots, l_{q-1}}^{e_{1}, \ldots, e_{r}}$. In fact, assume $\phi \neq 0$ and take an integer $k$, with $1 \leq k \leq r$ and $e_{k} \neq e_{1}$, then we have, for example, for some $k=k\left(e_{1}, \ldots, e_{r}\right)$ such that $e_{k} \neq e_{1}$, we can write as follows:

$$
\tilde{a}_{i_{1}, \ldots, l_{q-1}}^{e_{1}, \ldots, e_{r}}= \begin{cases}\left(e_{k}-e_{1}\right)^{-1} a_{i_{1}, \ldots, k, \ldots, l_{q-1}}^{e_{1}, \ldots, e_{r}} & k \notin\left\{i_{1}, \ldots, i_{q-1}\right\} \\ 0, & \text { otherwise. }\end{cases}
$$

Hence

$$
\limsup _{e_{1}+\cdots+e_{r} \rightarrow \infty} \sqrt[e_{1}+\cdots+e_{r}]{\left|\tilde{a}_{i_{1}, \ldots, l_{q-1}}^{e_{1}, \ldots, e_{r}}\right|}
$$

is finitely bounded, therefore $\sum \psi^{e_{1}, \ldots, e_{r}}$ converges in some neighborhood of $x$.

From Step 2, we have

$$
H^{q}\left(M_{e_{1}, \ldots, e_{r}}^{\bullet}\right)= \begin{cases}M_{e_{1}, \ldots, e_{r}}^{q}, & e_{1}=\cdots=e_{r}, \\ 0, & \text { otherwise. }\end{cases}
$$

From Step 1 and Step 3,

$$
\mathscr{H}^{q}\left(\omega_{X / Y}^{\bullet}\right)_{x}=\left\{\phi=\sum_{e_{1}, \ldots, e_{r}} \phi^{e_{1}, \ldots, e_{r}} \in \sum_{e_{1}, \ldots, e_{r}} H^{q}\left(M_{e_{1}, \ldots, e_{r}}^{\bullet}\right) ; \phi \text { converges }\right\} .
$$

Thus we have

$$
\mathscr{H}^{q}\left(\omega_{X / Y}^{\bullet}\right)_{x}=\left\{\sum_{1 \leq i_{1}<\cdots<i_{q} \leq r} a_{i_{1} \cdots l_{q}} f_{i_{1}} \wedge \cdots \wedge f_{i_{q}} ; a_{i_{1}, \ldots l_{q}} \in f^{-1} \mathcal{O}_{Y, x}\right\} / I
$$

as desired.

CASE 3: Let $x$ be the point $\left(0,0, \ldots, 0, t_{k+1}, \ldots, t_{r}\right)$ such that $t_{k+1} \cdots t_{r} \neq 0$ $(k<r-1)$, then $x \in X$ has an affine open neighborhood

$$
\text { Spec } C\left[z, t_{1}, \ldots, t_{k}, t_{k+1}^{ \pm 1}, \ldots, t_{r}^{ \pm 1}\right] /\left(z-t_{1} \cdots t_{r}\right) \text {. }
$$

We change coordinate $t_{1}$ by $T=t_{1} t_{k+1} \cdots t_{r}$. This change of coordinate induces an isomorphism of $\mathrm{fs} \log$ analytic spaces. We denote by $X^{\prime}$ the resulting open set:

$$
X^{\prime}=\left(\operatorname{Spec} C\left[z, T, t_{2}, \ldots, t_{k}, t_{k+1}^{ \pm 1}, \ldots, t_{r}^{ \pm 1}\right] /\left(z-T t_{2} \cdots t_{k}\right)\right)
$$

Then $f$ becomes

$$
f^{\prime}: X^{\prime} \rightarrow Y ; \quad\left(z, T, t_{2}, \ldots, t_{r}\right) \mapsto T t_{2} \cdots t_{k}
$$


Now we will compute the cohomology $\mathscr{H}^{q}\left(\omega_{X^{\prime} / Y}^{\bullet}\right)_{x}$. We have

$$
\omega_{X^{\prime} / Y, x}^{1}=\frac{\mathcal{O}_{X, x} f_{1}^{\prime} \oplus \cdots \oplus \mathcal{O}_{X, x} f_{r}^{\prime}}{\mathcal{O}_{X, x}\left(f_{1}^{\prime}+\cdots+f_{k}^{\prime}\right)}
$$

where $f_{1}^{\prime}=d T / T, f_{i}^{\prime}=d t_{i} / t_{i}$ for $i>1$. Similarly as Case 2, we define submodules $M_{e_{1}, \ldots, e_{r}}^{q}$ of $\omega_{X^{\prime} / Y, x}^{q}$. For

$$
\phi^{\prime}=\left(\sum_{2 \leq l_{1}<\cdots<l_{q} \leq r} a_{i_{1}, \ldots, l_{q}} f_{l_{1}}^{\prime} \wedge \cdots \wedge f_{l_{q}}^{\prime}\right) t_{1}^{e_{1}} \cdots t_{r}^{e_{r}} \in M_{e_{1}, \ldots, e_{r}}^{\prime q},
$$

we have

$$
\begin{aligned}
d \phi^{\prime}=t_{1}^{e_{1}} \cdots t_{r}^{e_{r}}\left(\sum_{2 \leq l_{1}<\cdots<l_{q} \leq r}\right. & \left(\sum_{\substack{j \neq 1 \\
J \notin\left\{1, l_{1}, \ldots, l_{q}\right\}}}^{k} a_{i_{1}, \ldots, l_{q}}\left(e_{j}-e_{1}\right) f_{j}^{\prime} \wedge f_{l_{1}}^{\prime} \wedge \cdots \wedge f_{l_{q}}^{\prime}\right. \\
& \left.\left.+\sum_{\substack{j=k+1 \\
j \notin\left\{1, l_{1}, \ldots, l_{q}\right\}}}^{r} a_{i_{1}, \ldots, l_{q}} e_{j} f_{J}^{\prime} \wedge f_{l_{1}}^{\prime} \wedge \cdots \wedge f_{l_{q}}^{\prime}\right)\right) .
\end{aligned}
$$

By a similar argument in Case 2 , we can show that $\mathscr{H}^{q}\left(\omega_{X / Y}^{\bullet}\right)_{x}$ is isomorphic to the stalk of the right hand side of (3.4) at $x$.

TheOREM A (Log relative Poincaré lemma). Let $f: X \rightarrow Y$ be a morphism of $f$ s log analytic spaces satisfying 3.2. Let $\omega_{X / Y}^{\cdot \log }$ be $\sigma^{*} \omega_{X / Y}^{\bullet}$, here $\sigma: X^{\log } \rightarrow X$ is the canonical map. Then the canonical morphism

$$
\left(f^{\log }\right)^{-1} \mathcal{O}_{Y}^{\log } \rightarrow \omega_{X / Y}^{\bullet \log }
$$

is a quasi-isomorphism.

Proof. Let $\left(P \rightarrow \mathscr{M}_{X}, Q \rightarrow \mathscr{M}_{Y}, Q \rightarrow P\right)$ be a chart of the morphism $f$. Let $S$ (resp. $T$ ) be Spec $C[P]_{\text {an }}$ (resp. Spec $C[Q]_{\text {an }}$ ). The question being local, we may assume $X \cong Y \times_{T} S$. Since $\omega_{X / Y}^{\bullet \log } \cong \omega_{S / T}^{\bullet \log } \otimes_{\mathcal{O}_{S}} \mathcal{O}_{X} \cong \omega_{S / T}^{\bullet \log } \otimes_{\mathcal{O}_{T}} \mathcal{O}_{Y}$, we have

$$
\mathscr{H}^{q}\left(\omega_{X / Y}^{\bullet \log }\right) \cong \mathscr{H}^{q}\left(\omega_{S / T}^{\bullet \log }\right) \otimes_{\mathcal{O}_{T}} \mathcal{O}_{Y} .
$$

On the other hand, as $\mathcal{O}_{Y}^{\log } \cong \mathcal{O}_{T}^{\log } \otimes \mathcal{O}_{T} \mathcal{O}_{Y}$, we may assume that $X=S$ and $Y=T$. Let $x^{\prime} \in X^{\log }, x=\sigma\left(x^{\prime}\right) \in X$ and $y=f(x) \in Y$. Let $\left(t_{i}\right)_{1 \leq \imath \leq r}$ be a family of elements of $\mathscr{M}_{X, x}^{\mathrm{gp}}$ whose classes in $\mathscr{M}_{X, x}^{\mathrm{gp}} / \mathcal{O}_{X, x}^{*}$ is a basis of $\mathscr{M}_{X, x}^{\mathrm{gp}} / \mathcal{O}_{X, x}^{*}$ over $\boldsymbol{Z}$ and $u$ an element of $\mathscr{M}_{Y, y}^{\mathrm{gp}}$ whose class in $\mathscr{M}_{Y, y}^{\mathrm{gp}} / \mathcal{O}_{Y, y}^{*}$ is a basis of $\mathscr{M}_{Y, y}^{\mathrm{gp}} / \mathcal{O}_{Y, y}^{*}$ over $\boldsymbol{Z}$.

StEP 1. Let $A$ (resp. $B$ ) be the polynomial ring $\mathcal{O}_{Y, y}[z]$ (resp. $\left.\mathcal{O}_{Y, y}\left[T_{1}, \ldots, T_{r}\right]\right)$. We define a morphism of $\mathcal{O}_{Y, y}$-algebras by 


$$
A \rightarrow B ; \quad z \mapsto T_{1}+\cdots+T_{r}
$$

and a morphism $\phi$ of complexes of $\mathcal{O}_{Y, y}$-modules by

$$
\phi: \Omega_{B / A}^{\bullet} \rightarrow \omega_{X / Y, x}^{\bullet \log } ; \quad d T_{\imath} \mapsto \frac{d t_{i}}{t_{i}} .
$$

Let $A \rightarrow \Omega_{B / A}^{\bullet}$ be the canonical morphism of complexes. Then the diagram

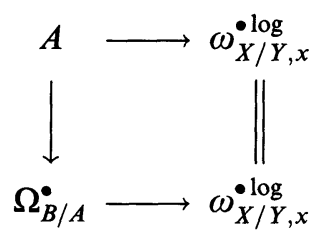

is commutative.

STEP 2. The morphism $A \rightarrow \Omega_{B / A}^{\bullet}$ is a quasi-isomorphism. This is well known.

STEP 3. We define increasing filtrations $F$ of $\Omega_{B / A}^{\bullet}$ and $G$ of $\omega_{X / Y, x}^{\bullet \log }$ by $F_{k}\left(\Omega_{B / A}^{q}\right):=\left\{\Sigma f \eta ; f \in B, \operatorname{deg} f \leq k, \eta=d T_{l_{1}} \wedge \cdots \wedge d T_{l_{q}} \in \Omega_{B / A}^{q}\left(i_{1}<\cdots<i_{q}\right)\right\}$, $G_{k}\left(\omega_{X / Y}^{q \log }\right):=$ the image of $\operatorname{fil}_{k}\left(\mathcal{O}_{X}^{\log }\right) \otimes \omega_{X / Y}^{q}$ in $\omega_{X / Y}^{q \log }$.

Here fil is the filtration introduced after 2.9. Since $\phi$ respects to filtrations $F$ and $G$, it induces $\operatorname{Gr}(\phi): \operatorname{Gr}_{k}^{F}\left(\Omega_{B / A}^{\bullet}\right) \rightarrow \operatorname{Gr}_{k}^{G}\left(\Omega_{X / Y}^{\bullet \log }\right)_{x}$. We claim that $\operatorname{Gr}(\phi)$ is a quasi-isomorphism.

(From Step 3, $\phi: \Omega_{B / A}^{\bullet} \rightarrow \omega_{X / Y, x}^{\bullet \log }$ is a quasi-isomorphism, hence $A \rightarrow \omega_{X / Y, x}^{\bullet \log }$ is a quasi-isomorphism.)

Now we prove Step 3. By 2.11, there is the canonical isomorphism of complexes

$$
\psi: \operatorname{Gr}_{k}^{G}\left(\omega_{X / Y}^{\bullet \log }\right) \cong \sigma^{-1}\left(\operatorname{Sym}_{Z}^{k}\left(\mathscr{M}_{X}^{\mathrm{gp}} / \mathcal{O}_{X}^{*}\right)\right) \otimes_{Z} \sigma^{-1} \omega_{X / Y}^{\bullet}
$$

Let

$$
\xi_{2}: H^{q}\left(\mathrm{Gr}_{k}^{F} \Omega_{B / A}^{\bullet}\right) \stackrel{\sim}{\rightarrow}\left(f^{-1} \mathcal{O}_{Y} \otimes \bigwedge^{q} \frac{\mathscr{M}_{X}^{\mathrm{gp}} / \mathcal{O}_{X}^{*}}{f^{-1}\left(\mathscr{M}_{Y}^{\mathrm{gp}} / \mathcal{O}_{Y}^{*}\right)} \otimes \operatorname{Sym}_{Z}^{k}\left(\mathscr{M}_{X}^{\mathrm{gp}} / \mathcal{O}_{X}^{*}\right)\right)_{y}
$$

be the natural isomorphism. Let $\xi_{1}$ be a morphism as in 3.3. Put $\xi=$ $\left(\xi_{1} \otimes \mathrm{id}\right) \circ \xi_{2}$. Then $\xi$ makes the following diagram commutative:

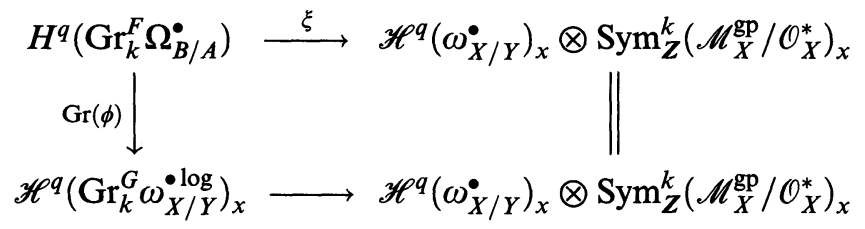

Thus we have $\operatorname{Gr}(\phi)$ is a quasi-isomorphism as desired. 
4. The morphism $\tau^{*} R f_{*} \omega_{X / Y}^{\bullet} \rightarrow R f_{*}^{\log C \otimes \mathcal{O}_{Y}^{\log }}$

Let $f: X \rightarrow Y$ be a proper smooth morphism of complex manifolds. Then we have a quai-isomorphism $R f_{*} C \otimes \otimes_{C} \mathcal{O}_{Y} \stackrel{\sim}{\rightarrow} R f_{*} \Omega_{X / Y}^{\bullet}$. We construct a similar quasi-isomorphism on fs log analytic spaces satisfying 3.2 .

LEMma 4.1 (Proper base change theorem). Let $X, Y, Z, W$ be locally compact Hausdorff topological spaces and $f: X \rightarrow Y, g: Z \rightarrow W, \sigma: X \rightarrow Z$, $\tau: Y \rightarrow W$ continuous maps such that the diagram

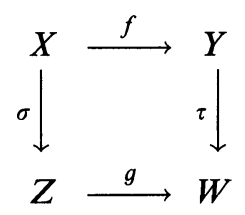

is cartesian. Assume that $g$ is proper (i.e., an inverse image of a compact set is compact). Then for any complex $K^{\bullet}$ of sheaves of abelian groups on $Z$, we have a quasi-isomorphism

$$
\tau^{-1} R g_{*} K^{\bullet} \stackrel{\sim}{\rightarrow} R f_{*}\left(\sigma^{-1} K^{\bullet}\right)
$$

See [SGA, p. 39].

LEMMA 4.2. Let $f: X \rightarrow Y$ be a proper continuous map of locally compact Hausdorff topological spaces, $\mathscr{A}$ a sheaf of rings on $Y, \mathscr{F}$ a sheaf of $\left(f^{-1} \mathscr{A}\right)$ modules on $X$ and $\mathscr{G}$ a sheaf of $\mathscr{A}$-modules such that $\mathscr{G}_{y}$ is a free $\mathscr{A}_{y}$-module for each $y \in Y$. Then we have a quasi-isomorphism

$$
R f_{*} \mathscr{F} \otimes \mathscr{A} \mathscr{G} \stackrel{\sim}{\rightarrow} R f_{*}\left(\mathscr{F} \otimes_{f^{-1} \mathscr{A}} f^{-1} \mathscr{G}\right)
$$

Proof. First notice that, using 4.1, we may assume that $Y$ is a point. Hence it is enough to prove that

$$
\oplus_{I} H^{m}(X, \mathscr{F}) \rightarrow H^{m}\left(X, \oplus_{I} \mathscr{F}\right)
$$

is an isomorphism for all $m$. If $I$ is a finite set, it is clear. If $I$ is an infinite set, use [Ive, p. 173, Theorem 5.1].

4.3. Let $X, Y, f$ be as in Theorem $\mathrm{A}$ in section 3 and moreover, assume that $f$ is proper. Let $\stackrel{\circ}{X}$ (resp. $\stackrel{\circ}{Y}$ ) be the underlying analytic space of $X$ (resp. $Y$ ). We have the canonical commutative diagram of topological spaces

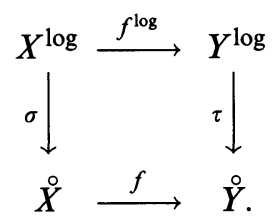


Let $X^{\prime}$ be a $\log$ analytic space $\left(\stackrel{\circ}{X}, f^{*} \mathscr{M}_{Y}\right)$. Let $g: X^{\prime \log } \rightarrow Y^{\log }, h: X^{\log } \rightarrow$ $X^{\prime \log }, \tilde{\sigma}: X^{\prime \log } \rightarrow \dot{X}$ be the canonical maps, respectively.

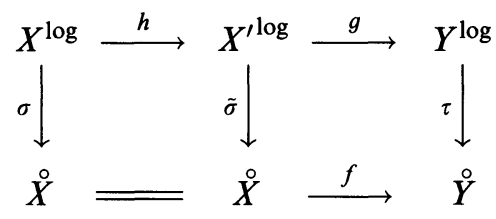

4.4. Let $\mathscr{F}$ be a locally free $\mathcal{O}_{X}$-module of finite rank. From the natural morphism $\mathscr{F} \rightarrow \sigma_{*} \sigma^{*} \mathscr{F} \cong \tilde{\sigma}_{*} h_{*} \sigma^{*} \mathscr{F}$, we have an associated morphism $\tilde{\sigma}^{*} \mathscr{F} \rightarrow$ $R h_{*} \sigma^{*} \mathscr{F}$.

LEMMA 4.5. $\quad \tilde{\sigma}^{*} \mathscr{F} \rightarrow R h_{*} \sigma^{*} \mathscr{F}$ is a quasi-isomorphism.

Proof. Since taking a cohomology commutes with taking a direct sum, our task is now to show $\tilde{\sigma}^{*} \mathcal{O}_{X} \rightarrow R h_{*} \sigma^{*} \mathcal{O}_{X}$ is a quasi-isomorphism. This is equivalent to show that $\mathcal{O}_{X^{\prime}}^{\log } \rightarrow R h_{*} \mathcal{O}_{X}^{\log }$ is a quasi-isomorphism. Let $x$ be a point of $X^{\prime \log }$. Since $h$ is proper, we have

$$
\left(R^{l} h_{*} \mathcal{O}_{X}^{\log }\right)_{x}=\boldsymbol{H}^{l}\left(h^{-1}(x), \mathcal{O}_{X}^{\log } \mid h^{-1}(x)\right) .
$$

Let $r$ be $\operatorname{rank}_{Z}\left(\mathscr{M}_{X, x}^{\mathrm{gp}} / \mathcal{O}_{X, x}^{*}\right)$. Then we have $h^{-1}(x) \cong\left(\boldsymbol{S}^{1}\right)^{r-1}$. Let $X_{k}$ be the $\log$ analytic space whose base space is $\stackrel{\circ}{X}$ and whose $\log$ structure is locally defined by the chart

$$
N^{k} \rightarrow \Gamma\left(X_{k}, \mathcal{O}_{X_{k}}\right)=\Gamma\left(X, \mathcal{O}_{X}\right) ; \quad\left(e_{1}, \ldots, e_{k}\right) \mapsto t_{1}^{e_{1}} \cdots t_{k-1}^{e_{k-1}} t_{k}^{e_{k}} \cdots t_{r}^{e_{k}}
$$

(Hence we have $X_{r}=X$ and $X_{1}=X^{\prime}$.) For $1 \leq k \leq r-1$, let $\psi_{k}: X_{k+1} \rightarrow X_{k}$ be the morphism of $\log$ analytic spaces, that is defined by the morphism of monoids

$$
N^{k} \rightarrow N^{k+1} ; \quad\left(e_{1}, \ldots, e_{k}\right) \mapsto\left(e_{1}, \ldots, e_{k}, e_{k}\right)
$$

Let $h_{k}: X_{k+1}^{\log } \rightarrow X_{k}^{\log }$ be the associated morphism to $\psi_{k}$. Then $h_{k}^{-1}(x) \cong \boldsymbol{S}^{1}$. Therefore, in order to show being a quasi-isomorphism $\mathcal{O}_{X^{\prime}}^{\log } \stackrel{\sim}{\rightarrow} R h_{*} \mathcal{O}_{X}^{\log }$, it is enough to prove that the following morphism are quasi-isomorphisms:

$$
\mathcal{O}_{X_{k}}^{\log } \stackrel{\sim}{\rightarrow} R h_{k *} \mathcal{O}_{X_{k+1}}^{\log } \quad(1 \leq k \leq r-1) .
$$

We will prove it only in the case $r=2$, since the argument is the same as in the general case. Set $h=h_{1}$. We compute the cohomology of $h^{-1}(x)=\boldsymbol{S}^{1}$ with coefficients in $\mathcal{O}_{X}^{\log } \mid h^{-1}(x)$ in the Cech method. We define a coordinate $\theta$ on $S^{1}$ defined by

$$
\boldsymbol{S}^{1}=\{\exp (\sqrt{-1} \theta) ; \theta \in \boldsymbol{R}\} .
$$

Let $\left\{U_{1}, U_{2}\right\}$ be an open covering of $\boldsymbol{S}^{1}$ defined by 


$$
\begin{aligned}
& U_{1}=\{\exp (\sqrt{-1} \theta) ; 0<\theta<2 \pi\}, \\
& U_{2}=\{\exp (\sqrt{-1} \theta) ; \pi<\theta<3 \pi\} .
\end{aligned}
$$

Let $V_{1}$ (resp. $\left.V_{2}\right)$ be an open set $\{\exp (\sqrt{-1} \theta) ; 0<\theta<\pi\}$ (resp. $\{\exp (\sqrt{-1} \theta)$; $\pi<\theta<2 \pi\})$ of $\boldsymbol{S}^{1}$. If $V$ be the intersection of $U_{1}$ and $U_{2}$, then $V$ is a disjoint union of $V_{1}$ and $V_{2}$. Since $\mathcal{O}_{X}^{\log }\left|U_{1}, \mathcal{O}_{X}^{\log }\right| U_{2}$ and $\mathcal{O}_{X}^{\log } \mid V$ are constant sheaves, we have

$$
\boldsymbol{H}^{k}\left(U_{i}, \mathcal{O}_{X}^{\log } \mid U_{i}\right)=\boldsymbol{H}^{k}\left(V, \mathcal{O}_{X}^{\log } \mid V\right)=0
$$

for $k>0$. Hence, we can compute the Čech cohomology of $\mathcal{O}_{X}^{\log } \mid h^{-1}(x)$ by the open covering $\left\{U_{1}, U_{2}\right\}$. Let $y=\tilde{\sigma}(x) \in X$. We denote the restriction of $\mathcal{O}_{X}^{\mathrm{log}}$ to $U_{1}$ (resp. $U_{2}$ ) by $\mathcal{O}_{X}^{\log } \mid U_{1}=\mathcal{O}_{X, y}\left[T_{1}, T_{2}\right]$ (resp. $\mathcal{O}_{X}^{\log } \mid U_{2}=\mathcal{O}_{X, y}\left[T_{1}^{\prime}, T_{2}^{\prime}\right]$ ) where $T_{l}, T_{l}^{\prime}$ are variables such that the difference of $T_{l}$ and $T_{l}^{\prime}$ on $V$ is in $2 \pi \sqrt{-1} Z$. From the assumption of $f$, we have $T_{1}+T_{2}=T_{1}^{\prime}+T_{2}^{\prime}$. Therefore we may assume that

$$
T_{1}^{\prime}=T_{1}+2 \pi \sqrt{-1}, \quad T_{2}^{\prime}=T_{2}-2 \pi \sqrt{-1} .
$$

Thus we have the following Čech complex $C^{\bullet}$

$$
\begin{gathered}
C^{0}=\mathcal{O}_{X, y}\left[T_{1}, T_{2}\right] \oplus \mathcal{O}_{X, y}\left[T_{1}^{\prime}, T_{2}^{\prime}\right], \\
C^{1}=\mathcal{O}_{X, y}\left[T_{1}, T_{2}\right] \oplus \mathcal{O}_{X, y}\left[T_{1}, T_{2}\right], \\
C^{l}=0 \quad(i \geq 2), \\
d: C^{0} \rightarrow C^{1} ;\left(p\left(T_{1}, T_{2}\right), q\left(T_{1}^{\prime}, T_{2}^{\prime}\right)\right) \\
\mapsto\left(p\left(T_{1}, T_{2}\right)-q\left(T_{1}, T_{2}\right), p\left(T_{1}, T_{2}\right)-q\left(T_{1}+2 \pi \sqrt{-1}, T_{2}-2 \pi \sqrt{-1}\right)\right) .
\end{gathered}
$$

Hence we have

$$
\begin{aligned}
\boldsymbol{H}^{0}\left(C^{\bullet}\right) & =\operatorname{ker} d \\
& =\left\{p\left(T_{1}, T_{2}\right) \in \mathcal{O}_{X, y}\left[T_{1}, T_{2}\right] ; p\left(T_{1}, T_{2}\right)=p\left(T_{1}+2 \pi \sqrt{-1}, T_{2}-2 \pi \sqrt{-1}\right)\right\} \\
& =\mathcal{O}_{X, y}\left[T_{1}+T_{2}\right] \cong \mathcal{O}_{X^{\prime}, x}^{\log } .
\end{aligned}
$$

It is clear that $\boldsymbol{H}^{1}\left(C^{\bullet}\right)=0$. This completes the proof.

Similarly, we have the following proposition.

Proposition 4.6. Let $X$ be an $f$ s log analytic space, $\mathscr{F}$ a locally free $\mathcal{O}_{X^{-}}$ module of finite rank and $\tau: X^{\log } \rightarrow X$ the canonical continuous map. Then we have a quasi-isomorphism

$$
\mathscr{F} \stackrel{\sim}{\rightarrow} R \tau_{*} \tau^{*} \mathscr{F}
$$

Hence

$$
\tau_{*} \mathcal{O}_{X}^{\log } \cong \mathcal{O}_{X}, \text { and } R^{l} \tau_{*} \mathcal{O}_{X}^{\log }=0, \quad \text { for } i \geq 1
$$


Proposition 4.7. Let $X, Y, f, \tau$ be as in 4.3. We have a quasi-isomorphism

$$
\tau^{*} R f_{*} \omega_{X / Y}^{\bullet} \stackrel{\sim}{\rightarrow} R f_{*}^{\log } \omega_{X / Y}^{\bullet \log }
$$

Proof. We have the notation in 4.3. From 2.7 (iii), the following diagram of topological spaces is catesian.

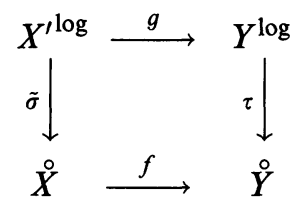

By 4.1 , we have a quasi-isomorphism $\tau^{-1} R f_{*} \omega_{X / Y}^{\bullet} \stackrel{\sim}{\rightarrow} R g_{*} \tilde{\sigma}^{-1} \omega_{X / Y}^{\bullet}$. Thus using 4.2, we have quasi-isomorphisms

$$
\begin{aligned}
\tau^{*} R f_{*} \omega_{X / Y}^{\bullet} & =\left(\tau^{-1} R f_{*} \omega_{X / Y}^{\bullet}\right) \otimes_{\tau^{-1} \mathcal{O}_{Y}} \mathcal{O}_{Y}^{\log } \\
& \stackrel{\sim}{\rightarrow} R g_{*}\left(\tilde{\sigma}^{-1} \omega_{X / Y}^{\bullet}\right) \otimes_{\tau^{-1} \mathcal{O}_{Y}} \mathcal{O}_{Y}^{\log } \\
& \stackrel{\sim}{\rightarrow} R g_{*}\left(\tilde{\sigma}^{-1} \omega_{X / Y}^{\bullet} \otimes_{(\tau g)^{-1} \mathcal{O}_{Y}} g^{-1} \mathcal{O}_{Y}\right) .
\end{aligned}
$$

Since $\mathcal{O}_{X^{\prime}}^{\log } \cong \tilde{\sigma}^{-1} \mathcal{O}_{X} \otimes_{(\tau g)^{-1}} g^{-1} \mathcal{O}_{Y}^{\log }$, we have a quasi-isomorphism

$$
\tau^{*} R f_{*} \omega_{X / Y}^{\bullet} \stackrel{\sim}{\rightarrow} R g_{*} \tilde{\sigma}^{*} \omega_{X / Y}^{\bullet}
$$

By 4.5 , we have a quasi-isomorphism $\tilde{\sigma}^{*} \omega_{X / Y}^{\bullet} \stackrel{\sim}{\rightarrow} R h_{*} \sigma^{*} \omega_{X / Y}^{\bullet} . \quad$ Since $f^{\log }=g h$, we obtain quasi-isomorphisms

$$
\begin{aligned}
R g_{*} \tilde{\sigma}^{*} \omega_{X / Y}^{\bullet} & \stackrel{\sim}{\rightarrow} R g_{*} R h_{*} \sigma^{*} \omega_{X / Y}^{\bullet} \\
& \stackrel{\sim}{\rightarrow} R f_{*}^{\log } \sigma^{*} \omega_{X / Y}^{\bullet} .
\end{aligned}
$$

By 4.8-4.10, we obtain the desired quasi-isomorphism.

THEOREM B. Let $f: X \rightarrow Y$ be a proper morphism of $f s$ log analytic spaces that satisfies 3.2. Then we have a quasi-isomorphism

$$
R f_{*}^{\log } C \otimes{ }_{C} \mathcal{O}_{Y}^{\log } \cong \tau^{*} R f_{*} \omega_{X / Y}^{\bullet}
$$

Proof. By Theorem A, we have a quasi-isomorphism

$$
C \otimes_{C} f^{\log -1} \mathcal{O}_{Y}^{\log } \stackrel{\sim}{\rightarrow} \omega_{X / Y}^{\bullet \log }
$$

Hence, using 4.2, we have quasi-isomorphisms

$$
\begin{aligned}
R f_{*}^{\log C} \otimes \otimes_{C} \mathcal{O}_{Y}^{\log } & \stackrel{\sim}{\rightarrow} R f_{*}^{\log }\left(C \otimes_{C} f^{\log -1} \mathcal{O}_{Y}^{\log }\right) \\
& \stackrel{\sim}{\rightarrow} R f_{*}^{\log }\left(\omega_{X / Y}^{\bullet \log }\right) .
\end{aligned}
$$

Theorem B follows from 4.7. 


\section{Log Hodge structures}

The aim of this section is to prove Theorem C. A log Hodge structure in Theorem $\mathrm{C}$ is a $\log$ geometric interpretation of object called a limit mixed Hodge structure in [St1].

Let $X$ be an fs $\log$ analytic space. For $x \in X$, let $\mathscr{Y}_{x}$ (resp. $\left.\overline{\mathscr{Y}}_{x}\right)$ be the set of all homomorphisms $\mathscr{M}_{X, x}^{\mathrm{gp}} \rightarrow \boldsymbol{R}_{>0}$ (resp. $\mathscr{M}_{X, x} \rightarrow \boldsymbol{R}_{\geq 0}$ ) which are extensions of

$$
\mathcal{O}_{X, x}^{*} \rightarrow \boldsymbol{R}_{>0} ; \quad f \mapsto|f(x)| .
$$

We introduce on $\mathscr{Y}_{x}$ (resp. $\overline{\mathscr{Y}}_{x}$ ) the topology in the following way. If $a_{1}, \ldots, a_{r}$ are elements of $\mathscr{M}_{X, x}^{\mathrm{gp}}$ (resp. $\mathscr{M}_{X, x}$ ) whose classes in $\mathscr{M}_{X, x}^{\mathrm{gp}} / \mathcal{O}_{X, x}^{*}$ (resp. $\mathscr{M}_{X, x} / \mathcal{O}_{X, x}^{*}$ ) generate $\mathscr{M}_{X, x}^{\mathrm{gp}} / \mathcal{O}_{X, x}^{*}$ as a group (resp. $\mathscr{M}_{X, x} / \mathcal{O}_{X, x}^{*}$ as a monoid), $\mathscr{Y}_{x}$ (resp. $\overline{\mathscr{Y}}_{x}$ ) has the topology as a subspace of $\left(\boldsymbol{R}_{>0}\right)^{r}$ (resp. $\left.\left(\boldsymbol{R}_{\geq 0}\right)^{r}\right)$ in which $\mathscr{Y}_{x}$ (resp. $\left.\overline{\mathscr{Y}}_{x}\right)$ is embedded by $\psi \rightarrow\left(\psi\left(a_{i}\right)\right)_{1 \leq \imath \leq r}$.

We regard $\mathscr{Y}_{x}$ as a subspace of $\overline{\mathscr{Y}}_{x}$ by the fact that a homomorphism $\mathscr{M}_{X, x}^{\mathrm{gp}} \rightarrow \boldsymbol{R}_{>0}$ is uniquely induced to one $\mathscr{M}_{X, x} \rightarrow \mathscr{M}_{X, x}^{\mathrm{gp}} \rightarrow \boldsymbol{R}_{>0} \hookrightarrow \boldsymbol{R}_{\geq 0}$. Let $\xi_{x}$ be the element of $\overline{\mathscr{Y}}_{x}$ that sends $\mathscr{M}_{X, x}^{\mathrm{gp}}-\mathcal{O}_{X, x}^{*}$ to $0 \in \boldsymbol{R}_{\geq 0}$.

Lemma 5.1 (K. Kato). Let $y \in X^{\log }$ and let $x=\tau(y) \in X$. Let $\mathscr{Y}_{x, y}$ be the set of homomorphisms $\phi: \mathcal{O}_{X, y}^{\log } \rightarrow C$ having the following properties:

(1) $\phi$ is an extension of $\mathcal{O}_{X, x} \rightarrow C ; f \mapsto f(x)$.

(2) The composite $\mathscr{L}_{y} \hookrightarrow \mathcal{O}_{X, y}^{\log } \stackrel{\phi}{\rightarrow} \boldsymbol{C} \rightarrow \boldsymbol{C} / \boldsymbol{R}=\boldsymbol{R} \sqrt{-1}$ coincides with $\theta_{y}$ in 2.8 . Then there exists a unique bijection

$$
\mathscr{Y}_{x} \rightarrow \mathscr{Y}_{x, y} ; \quad \psi \mapsto \psi_{y}
$$

satisfying

$$
\psi(\exp (a))=\left|\exp \left(\psi_{y}(a)\right)\right|, \quad \text { for } a \in \mathscr{L}_{y} .
$$

Proof. Let $\eta=\operatorname{Re}\left(\psi_{y}\right)$. Then $\psi_{y}$ is uniquely determined by $\eta$. Let $t_{1}, \ldots, t_{r}$ be a family of elements of $\mathscr{L}_{y}$ whose image under exp is $Z$-basis of $\mathscr{M}_{X, x}^{\mathrm{gp}} / \mathcal{O}_{X, x}^{*} . \quad \eta\left(\right.$ resp. $\psi$ ) is uniquely determined by the image of $t_{1}, \ldots, t_{r}$ (resp. $\left.\exp \left(t_{1}\right), \ldots, \exp \left(t_{r}\right)\right)$. Put $\eta\left(t_{i}\right)=\log \left(\psi\left(\exp \left(t_{i}\right)\right)\right)$. Then we have the desired bijection.

We assume that the fs $\log$ analytic space $X$ satisfies the following condition:

5.2. Locally on $X$, there is an fs monoid $P$ and an ideal $\Sigma$ of $P$ such that $X$ is an open subspace of $(\operatorname{Spec} C[P] / \Sigma)_{a n}$ that is endowed with the log structure associated to $P \rightarrow C[P] / \Sigma$.

Definition 5.3 (K. Kato). Let $X$ be an fs log analytic space satisfying the condition 5.2. For $n \in Z$, a $\log$ Hodge structure $(\log$ HS) $\mathscr{H}$ on $X$ of weight $n$ is a triplet $\left(\mathscr{H}_{\boldsymbol{Q}}, \mathscr{H}_{\mathcal{O}}, l_{\mathscr{H}}\right)$ consisting of 
- a sheaf of $\boldsymbol{Q}$-modules $\mathscr{H}_{Q}$ on $X^{\log }$,

- a sheaf of $\mathscr{O}_{X}$-modules $\mathscr{H}_{\mathcal{O}}$ on $X$ endowed with a descending filtration $\left(\boldsymbol{F}^{l} \mathscr{H}_{\mathcal{O}}\right)_{i \in \boldsymbol{Z}}$ and with an integrable connection

$$
\nabla: \mathscr{H}_{\mathcal{O}} \rightarrow \omega_{X}^{1} \otimes_{\mathcal{O}_{X}} \mathscr{H}_{\mathcal{O}}
$$

- an isomorphism of $\mathcal{O}_{X}^{\log }$-modules

$$
l_{\mathscr{H}}: \mathscr{H}_{\boldsymbol{Q}} \otimes_{\boldsymbol{Q}} \mathcal{O}_{X}^{\log } \cong \tau^{*} \mathscr{H}_{\mathcal{O}}
$$

that satisfy the following conditions 5.4-5.9: module.

5.4. $\mathscr{H}_{\boldsymbol{Q}}$ is locally constant, and each stalk is free of finite rank as a $\boldsymbol{Q}$ -

5.5. $\mathscr{H}_{\mathcal{O}}$ is locally free of finite rank as an $\mathcal{O}_{X}$-module.

5.6. $\boldsymbol{F}^{\imath} \mathscr{H}_{\mathcal{O}}=\mathscr{H}_{\mathcal{O}}$ if $i \ll 0, \boldsymbol{F}^{\imath} \mathscr{H}_{\mathcal{O}}=0$ if $i \gg 0$.

5.7. Each $\boldsymbol{F}^{l} \mathscr{H}_{\mathcal{O}}$ is an $\mathcal{O}_{X}$-submodule of $\mathscr{H}_{\mathcal{O}}$, and is locally an $\mathcal{O}_{X}$-direct summand of $\mathscr{H}_{\mathfrak{O}}$.

5.8. $\nabla\left(\boldsymbol{F}^{l} \mathscr{H}_{\mathcal{O}}\right) \subset \omega_{X}^{1} \otimes_{\mathcal{O}_{X}} \boldsymbol{F}^{l-1} \mathscr{H}_{\mathcal{O}}$ for each $i$.

5.9. Let $x \in X$. Then there exists an open neighborhood $V$ of $\xi_{x}$ in $\overline{\mathscr{Y}}_{x}$ such that for any $y \in \tau^{-1}(x)$ and $\psi \in \mathscr{Y}_{x} \cap V, \mathscr{H}_{\boldsymbol{Q}, y}$ with the filtration $\boldsymbol{C} \otimes_{\mathcal{O}_{X, x}} \boldsymbol{F}^{l} \mathscr{H}_{\mathcal{O}, x}$ on $\boldsymbol{C} \otimes \mathscr{H}_{\boldsymbol{Q}, y}=\boldsymbol{C} \otimes_{\mathcal{O}_{X, x}} \mathscr{H}_{\mathcal{O}, x}$, the equality given by $\psi_{y}$, is a Hodge structure of weight $n$ in the classical sense. Here $\mathcal{O}_{X, x} \rightarrow C$ is $f \mapsto f(x)$.

Let $Y=\{z \in C|| z \mid<1\}$ be the unit disk with the log structure defined by the origin, $Y^{*}$ the punctured disk. Via the mapping $U \rightarrow Y^{*} ; u \mapsto \exp (2 \pi i u)$, the upper half plane $U=\{u \in C \mid \operatorname{Im}(u)>0\}$ becomes the universal covering of $Y^{*}$. The fundamental group $\pi_{1}\left(Y^{*}\right)=\pi_{1}\left(Y^{\log }\right)$ is generated by the translation $u \mapsto u+1$. Consider the subsheaf $Q[u] \subset \mathcal{O}_{Y}^{\log }$. Let $\sigma$ be the monodromy of $Q[u]$ around the origin. Then we have $\sigma: u \mapsto u-1$ and $\log \sigma=-d / d u$.

Lemma 5.10. Let $V$ be a Q-vector space, $N: V \rightarrow V$ nilpotent homomorphism and $Q[u]$ a polynomial ring in one variable over $Q$. We define the endomorphism $\Delta$ of $V \otimes_{Q} Q[u]$ to be $N \otimes 1-1 \otimes d / d u$. Then

$$
\operatorname{ker} \Delta=W:=\left\{\sum_{m=0}^{\infty} \frac{N^{m}(x)}{m !} \otimes u^{m} ; x \in V\right\} .
$$

Proof. Let $f$ be an element of $V \otimes Q[u]$. We can write $f=\sum_{l=0}^{m} x_{l} \otimes u^{l}$, $x_{\imath} \in V$. Then we have 


$$
\Delta(f)=\sum_{l=0}^{m-1}\left\{N x_{l}-(i+1) x_{\imath+1}\right\} \otimes u^{l}+N x_{m} \otimes u^{m} .
$$

Hence, $f \in \operatorname{ker} \Delta$ implies $x_{l}=N^{\imath}\left(x_{0}\right) / i$ ! for $(i \geq 1)$, therefore $f \in W . \quad$ It is clear that $W \subset \operatorname{ker} \Delta$.

LEMMA 5.11. Let $X$ be the analytic space Spec $C_{\text {an }}$ endowed with the log structure associated to $\boldsymbol{N} \rightarrow \boldsymbol{C} ; n \mapsto 0^{n}$ and $\mathscr{F}$ a locally constant sheaf of $Q$-vector spaces on the topological space $X^{\log }$. Let $t$ be a section of the sheaf of monoids on $X$ associated to its log structure such that $t$ is an image of $1 \in N$, and consider the subsheaf $\boldsymbol{Q}[u] \subset \mathcal{O}_{X}^{\log }$ where $u=(2 \pi \sqrt{-1})^{-1} \log t$. Let $N$ be the logarithm of the monodromy of $\mathscr{F}$. Assume $N$ is nilpotent. Then the restriction map of the sheaf $\mathscr{F} \otimes{ }_{Q} Q[u]$

$$
\Gamma\left(X^{\log }, \mathscr{F} \otimes Q[u]\right) \rightarrow \mathscr{F}_{\alpha} \otimes Q[u], \quad\left(\alpha \in X^{\log }\right)
$$

factors through the submodule

$$
\exp (u N) \mathscr{F}_{\alpha}:=\left\{\sum_{n=0}^{\infty} \frac{N^{n}(x)}{n !} \otimes u^{n} ; x \in \mathscr{F}_{\alpha}\right\} \subset \mathscr{F}_{\alpha} \otimes Q[u]
$$

and moreover, $\Gamma\left(X^{\log }, \mathscr{F} \otimes Q[u]\right) \rightarrow \exp (u N) \mathscr{F}_{\alpha}$ is an isomorphism.

Proof. Let $\Delta$ be $N \otimes 1-1 \otimes d / d u$. Since $\Delta$ is the logarithm of the monodromy of $\mathscr{F} \otimes Q[u]$, we have $\Gamma\left(X^{\log }, \mathscr{F} \otimes Q[u]\right) \stackrel{\sim}{\rightarrow} \operatorname{ker} \Delta \subset \mathscr{F} \otimes Q[u]$. From 5.10 , we have the desired isomorphism $\Gamma\left(X^{\log }, \mathscr{F} \otimes Q[u]\right) \stackrel{\sim}{\rightarrow} \exp (u N) \mathscr{F}_{\alpha}$.

5.12. Let $T$ be a topological space and $\mathscr{F}$ a sheaf on $T$. For a subset $S$ of $T$, we omit $\Gamma\left(S,\left.\mathscr{F}\right|_{S}\right)$ as $\Gamma(S, \mathscr{F})$.

Proposition 5.13 (F. Kato). Let $Y$ be a unit disk with the log structure defined by the origin and $f: X \rightarrow Y$ a proper morphism of $f_{s} \log$ analytic spaces that satisfies 3.2. Let $D$ be $f^{-1}(0)$ and $\tilde{X}^{*}$ the fibre product of $X$ and the universal covering of $Y^{*}$ over $Y^{*}$. Let $\tau: Y^{\log } \rightarrow Y$ be the canonical map. For $\alpha \in \tau^{-1}(0)$, we have Q)).

(i) $p: H^{m}\left(\left(f^{\log }\right)^{-1}(\alpha), C\right) \stackrel{\sim}{\rightarrow} H^{m}\left(\tilde{X}^{*}, C\right)\left(\right.$ resp. $H^{m}\left(\left(f^{\log }\right)^{-1}(\alpha), Q\right) \stackrel{\sim}{\rightarrow} H^{m}\left(\tilde{X}^{*}\right.$,

(ii) $\Gamma\left(\tau^{-1}(0), R^{m} f_{*}^{\log } \boldsymbol{C} \otimes \mathcal{O}_{Y}^{\log }\right) \stackrel{\sim}{\rightarrow} H^{m}\left(\left(f^{\log }\right)^{-1}(\alpha), C\right)\left(\right.$ resp. $\Gamma\left(\tau^{-1}(0), R^{m} f_{*}^{\log } \boldsymbol{Q}\right.$ $\left.\otimes \boldsymbol{Q}[u]) \stackrel{\sim}{\rightarrow} H^{m}\left(\left(f^{\log }\right)^{-1}(\alpha), Q\right)\right)$.

(iii) Let $\imath$ be a morphism as in Theorem $B$. Taking $\Gamma\left(\tau^{-1}(0)\right.$, ) on $\imath$, we got an isomorphism $q: H^{m}\left(D, \omega_{D}^{\bullet}\right) \stackrel{\sim}{\rightarrow} H^{m}\left(\left(f^{\log }\right)^{-1}(\alpha), C\right)$. Then the composite map $p \circ q$ is the same isomorphism as $[S t 1,(2.16)]$.

Proof. See [Usu] and [FKa, pp. 21-22]. 
5.14. let $Y:=\{z \in C|| z \mid<1\}$ be the unit disk, and $f: X \rightarrow Y$ a projective surjective morphism of complex manifolds. We assume that $f$ is smooth over the punctured disk $Y^{*}=Y-\{0\}$ and that $X_{0}=f^{-1}(0)$ is a reduced divisor with normal crossings. Let $P \in X_{0}$. We assume that there exists a coordinate neighborhood $U$ of $P$ with coordinates $\left(z_{0}, \ldots, z_{n}\right)$ and an integer $r$ with $1 \leq r \leq n$ such that $P=(0, \ldots, 0)$ and $f \mid U\left(z_{1}, \ldots, z_{n}\right)=z_{1} \cdots z_{r}=z$. Let $\mathscr{M}_{Y}$ (resp. $\mathscr{M}_{X}$ ) be a sheaf of holomorphic functions on $Y($ resp. $X)$ which are invertible outside the origin (resp. $X_{0}$ ).

THEOREM 5.15 (Usui). Let $f: X \rightarrow Y$ a morphism of $f s$ log analytic spaces

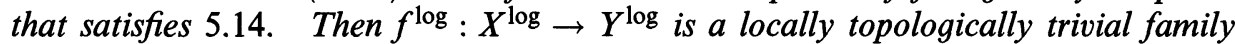
over the base. Moreover $R^{m} f_{*}^{\log Q}$ is a locally constant sheaf. (This is a special case of [Usu, Theorem 3.4].)

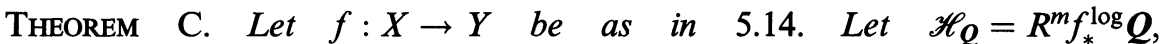
$\mathscr{H}_{\mathcal{O}}=R^{m} f_{*} \omega_{X / Y}^{\bullet}$ endowed with a filtration $\mathscr{F}^{l}:=R^{m} f_{*} \omega_{X / Y}^{\bullet \geq l}$ and $l$ the isomorphism as in Theorem $B$. Then the triplet $\left(\mathscr{H}_{\mathbf{Q}}, \mathscr{H}_{\mathcal{O}}, l\right)$ is a log Hodge structure on $Y$.

Proof. To show Theorem C, we will verify the conditions from 5.4 to 5.9. It is well known that the pair $\left(\mathscr{H}_{\mathcal{O}}, \mathscr{F}^{\bullet}\right)$ satisfies from 5.5 to 5.8 . 5.4 is direct from 5.15. Let $y \in Y$ be a smooth point, then it is well known that 5.9 is satisfied for $y$ from the theory of variation of Hodge structure. We verify 5.9 for the origin $y$ of $Y$ as follows. Let $w \in \tau^{-1}(y) \subset Y^{\log }$ and $u^{\prime}$ an element of $\mathscr{L}_{w}$ whose image under exp is the $Z$-basis of $\mathscr{M}_{Y, y}^{\mathrm{gp}} / \mathcal{O}_{Y, y}^{*}$, i.e., $\exp \left(u^{\prime}\right)=$ $\exp (2 \pi i u)=z$. Let $\psi_{w}: \mathcal{O}_{Y, w}^{\log } \rightarrow \boldsymbol{C}$ be an element of $\mathscr{Y}_{y, w}$ such that $\psi_{w}\left(u^{\prime}\right)=a$, $\psi: \mathscr{M}_{Y, y}^{\mathrm{gp}} \rightarrow \boldsymbol{R}_{>0}$ the corresponding element of $\mathscr{Y}_{y}$. We have the following commutative diagram

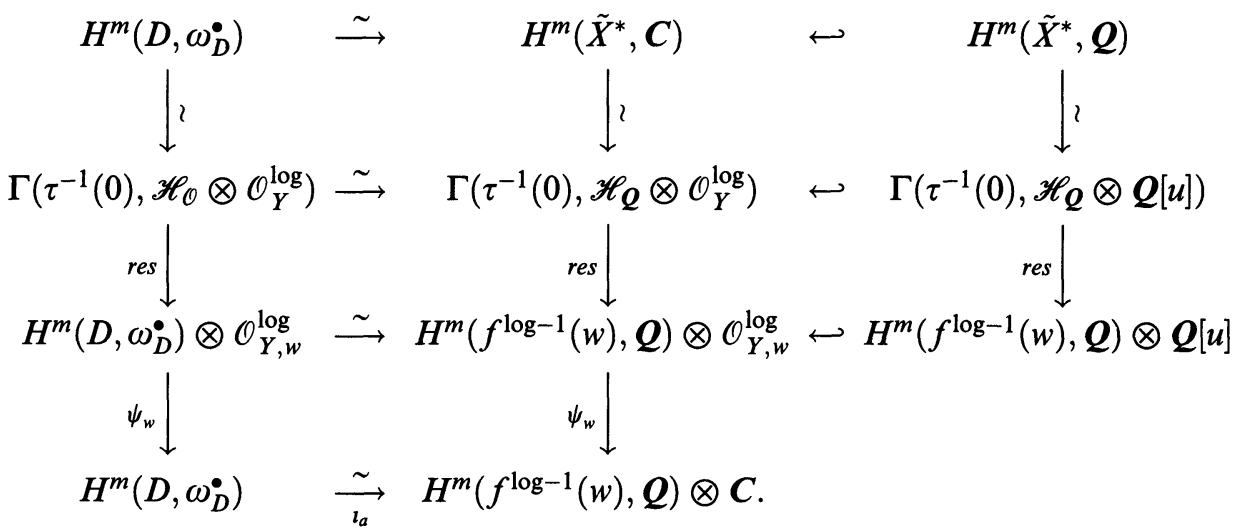

Here res is a restriction map. By 5.11, the image of $H^{m}\left(\tilde{X}^{*}, Q\right)$ in $H^{m}\left(f^{\log -1}(w), \boldsymbol{Q}\right) \otimes \mathcal{O}_{Y, w}^{\log }$ at the above diagram is $\exp (u N) \mathscr{H}_{\boldsymbol{Q}, \alpha}$. We have $\psi_{a} \circ$ res is the identity map. Hence an image of $H^{m}\left(\tilde{X}^{*}, Q\right)$ in the left hand side of $\boldsymbol{l}_{a}$ at the diagram is canonical. Consider $H^{m}\left(\tilde{X}^{*}, Q\right)$ as a submodule of 
$H^{m}\left(D, \omega_{D}^{\bullet}\right)$ in this way. Since the above diagram is commutative, the image of $H^{m}\left(X^{*}, \boldsymbol{Q}\right)$ by $l_{a}$ is $\exp ((a / 2 \pi i) N) \mathscr{H}_{\boldsymbol{Q}, w}$. Let $-($ resp. Z) be the complex conjugation mapping associated to the $\boldsymbol{Q}$-structure $H^{m}\left(\tilde{X}^{*}, \boldsymbol{Q}\right)$ (resp. $\left.\mathscr{H}_{\boldsymbol{Q}, w}\right)$. Then we have $==\exp (-(a / 2 \pi i) N) \circ-\circ \exp ((a / 2 \pi i) N)$. Hence

$$
\begin{aligned}
\mathscr{F}^{\bullet} \oplus \overline{\overline{\mathscr{F}}} & \left.=\mathscr{F}^{\bullet} \oplus \exp \left(-\frac{a}{2 \pi i} N\right) \overline{\exp \left(\frac{a}{2 \pi i} N^{\mathscr{F}} \bullet\right.}\right) \\
& \cong \exp \left(\frac{a}{2 \pi i} N\right) \mathscr{F}^{\bullet} \oplus \overline{\exp \left(\frac{a}{2 \pi i} N\right) \mathscr{F}^{\bullet}}
\end{aligned}
$$

By nilpotent orbit theorem [Sch, (4.9)], $\left(H^{m}\left(\tilde{X}^{*}, Q\right), H^{m}\left(D, \mathscr{W}_{D}^{\bullet}\right), \quad \exp ((a)\right.$ $\left.2 \pi i) N) \mathscr{F}^{\bullet}\right)$ is a Hodge structure if $\operatorname{Im}(a / 2 \pi i) \gg 0$. This is equivalent to say that $\left(\mathscr{H}_{\boldsymbol{Q}, w} \otimes \boldsymbol{C}, \mathscr{H}_{\boldsymbol{Q}, w}, \mathscr{F}^{\bullet}\right)$ is a Hodge structure if $\psi(z) \ll 0$.

\section{REFERENCES}

[Del] Deligne, P., Equations Différentiells á Points Singuliers Reguliers, Lecture Notes in Math., 163, Springer, 1970.

[De2] Deligne, P., Théorie de Hodge II, Inst. Hautes Études Sc1. Publ. Math., 40 (1971), 5-57.

[SGA] Deligne, P., Cohomologie Etale, Séminaire de Géométrie Algébrique du Boıs-Marie SGA

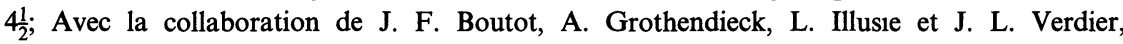
Lecture Notes in Math., 569, Springer, 1977.

[Gri] GrIfFiths, P., Topics in Transcendental Algebraic Geometry, Ann. of Math. Studies, Princeton Univ. Press, 1984.

[Ive] IVERSEN, B., Cohomology of Sheaves, Springer-Verlag, 1986.

[FKa] Kato, F., The relative log Poincaré lemma and relative log de Rham theory, Duke Math. J., 93 (1998), 179-206.

[Kal] KATo, K., Logarithmic structures of Fontaine-Illusie, Algebraic Analysis, Geometry, and Number Theory, Igusa, J.-I. ed., Johns Hopkıns Univ. Press, 1989, 191-224.

[Ka2] Kato, K., Problems concerning log Hodge structures, preprint.

[KN] Kato, K. AND NaKaYAMA, C., Log Betti cohomology, log etale cohomology, and log de Rham cohomology of $\log$ schemes over $C$, preprint.

[Sch] ScHmID, W., Variation of Hodge structure: The singularities of the period mapping, Invent. Math., 22 (1973), 211-319.

[St1] SteEnbrinK, J., Limits of Hodge structures, Invent. Math., 31 (1976), 229-257.

[St2] SteENBRINK, J., Logarithmic embeddings of varieties with normal crossings and mixed Hodge structures, Math. Ann., 301 (1995), 105-118.

[Usu] UsuI, S., Recovery of vanishing cycles by log geometry, preprint.

Graduate School of Mathematical Science

THE UNIVERSITY OF TOKYO

3-8-1 Komaba, Meguro-Ku, ToKyo

153-8914 JAPAN

E-mail: matubara@ms.u-tokyo.ac.jp 